\title{
The value of preoperative lymphocytes-to-monocytes ratio in predicting lymph node metastasis in gastric cancer
}

\author{
Dexiao Du ${ }^{1 \#}$, Ziliang Han ${ }^{2 \#}$, Dongbo Lian ${ }^{1}$, Buhe Amin $^{1}$, Wei Yan ${ }^{1}$, Nengwei Zhang ${ }^{1}$ \\ ${ }^{1}$ Department of General Surgery, Beijing Shijitan Hospital, Capital Medical University, Beijing 100038, China; ${ }^{2}$ Department of Gastrointestinal \\ Surgery, People's Hospital of Lixin County, Bozhou 236700, China \\ Contributions: (I) Conception and design: D Du, Z Han, N Zhang; (II) Administrative support: N Zhang; (III) Provision of study materials or patients: \\ N Zhang; (IV) Collection and assembly of data: D Du, Z Han, D Lian, B Amin; (V) Data analysis and interpretation: D Du, B Amin, W Yan; (VI) \\ Manuscript writing: All authors; (VII) Final approval of manuscript: All authors. \\ "These authors contributed equally to this work. \\ Correspondence to: Nengwei Zhang. Department of General Surgery, Beijing Shijitan Hospital of Capital Medical University, No.10 Tieyi Road, \\ Haidian District, Beijing 100038, China. Email: zhangnengweiw@163.com.
}

\begin{abstract}
Background: This study was aimed to evaluate value of preoperative lymphocytes-to-monocytes ratio (LMR) as a predictor for lymph node metastasis in gastric cancer.

Methods: Patients with pathologically diagnosed malignant gastric tumor were reviewed retrospectively. Patients' demographic data, clinical manifestations and LMR were collected. The postoperative pathology data including the tumor size, tumor sites, differentiation degree of tumor, depth of tumor invasion, metastatic lymph nodes, total dissected lymph nodes, distant metastasis, vessel carcinoma embolus, neural invasion, Lauren's classification, Ki-67 and TNM stage were collected. Receiver operating characteristic curve (ROC) was used to determine the cut-off point of LMR. The association between LMR and the clinicopathologic factors was further analyzed.

Results: A total of 177 subjects were enrolled into the study. LMR $=3.79$ was used as cut-off point; the patients with LMR $\geq 3.79$ were classified as high-LMR group (123 cases) and the patients with LMR $<3.79$ as low-LMR group (54 cases). LMR was an independent risk factor for lymph node metastasis.

Conclusions: Low preoperative LMR was positively correlated with lymph node metastasis in gastric cancer patients, and had predictive value for lymph node metastasis in gastric cancer patients.
\end{abstract}

Keywords: Gastric cancer; lymphocyte-to-monocyte ratio (LMR); lymph node metastasis

Submitted Mar 30, 2019. Accepted for publication Sep 11, 2019.

doi: $10.21037 /$ tcr.2019.09.17

View this article at: http://dx.doi.org/10.21037/tcr.2019.09.17

\section{Introduction}

Stomach cancer is the fifth most-common cancer in the world and the number of stomach cancer-resulted death was 700,000 in 2012, making it the third-leading cause of cancer-related death (1). In many countries and regions, due to lack of screening, the 5 -year survival rate of gastric cancer was only $20 \%$ and was $10-15 \%$ in advanced gastric cancer $(2,3)$. Lymph node metastasis is associated with the prognosis of gastric cancer and has been acknowledged as an independent indicator of poor prognosis $(4,5)$. There is no obvious symptoms in the early stage, however, lymph node metastasis usually has occurred when stomach cancer is diagnosed. Therefore, it is critical to make an early and accurate prediction of lymph node metastasis for the diagnosis of gastric cancer, staging, treatment and prognosis.

At present, tumor node metastasis (TNM) is widely used for gastric cancer staging. However, the lymph node involvement can only be determined pathologically after surgery. The magnetic resonance imaging (MRI) and computed tomography (CT), endoscopic ultrasound (EUS) 
and positron emission tomography computed tomography (PET-CT) were commonly used for preoperative evaluation of lymph node metastasis. But those methods still have disadvantages in accuracy and consistency (6,7). As reported, some protein markers have potential to indicate the lymph node metastasis of gastric cancer $(8,9)$, but those biomarkers are under laboratory research. Therefore, it is critical to find effective methods for assessing lymph node metastasis before surgery.

It is well known that inflammatory reactions play an important role in the process of tumor development, progression, invasion and metastatic prognosis (10). Systemic inflammatory response can inhibit normal apoptosis of senile cells and promote tumor progression, metastasis and diffusion by secreting inflammation factors and cytokines (11). LMR was reported to have the potential to reflect the degree of systemic inflammatory response and be related to the prognosis of gastric cancer $(12,13)$. As far as we know, there are few studies concerning the correlation between LMR and lymph node metastasis of gastric cancer. Therefore, we conducted this study to explore the association between LMR and the clinicopathologic factors, and to determine if LMR is a preoperative predictor for lymph node metastasis in patients with gastric cancer.

\section{Methods}

\section{Study subjects}

The patients with malignant gastric tumor, who received treatment in the department of general surgery, Beijing Shijitan Hospital of Capital Medical University from January 2014 to May 2017, were retrospectively reviewed. The inclusion criteria were patients with pathologicallydiagnosed malignant gastric tumor, patients who received surgical treatment and lymph node dissection, and patients without obvious infection, hemorrhage or other hematological disease. The exclusion criteria were patients who had received radiotherapy, chemotherapy, immunosuppressive therapy or other anti-tumor therapy, number of dissected lymph nodes $<15$, and multiple primary malignant neoplasm. This study was approved by the ethic committee of Beijing Shijitan Hospital of Capital Medical University.

\section{Data collection}

Patients' demographic data were collected. Peripheral venous blood samples were collected and the indexes including lymphocytes, monocytes, CA19-9 and CEA were detected within one week preoperatively. The postoperative pathology was independently analyzed by two experienced pathologists according to Japanese gastric cancer guidelines (14). The following data were collected, including tumor size, tumor sites, differentiation degree of tumor, depth of tumor invasion, metastatic lymph nodes, total dissected lymph nodes, distant metastasis, vessel carcinoma embolus, neural invasion, Lauren's classification, Ki-67 and TNM stage. LMR, percentage of lymph node metastasis and degree of lymphatic metastasis were calculated. Highly differentiated cancers included papillary adenocarcinoma and tubular adenocarcinoma, while highly undifferentiated cancers included signet ring cell carcinoma, mucinous adenocarcinoma and undifferentiated carcinoma. Patients with multiple pathological types of gastric cancer were subject to the least differentiated components. In the patients with multiple pathological types, the least differentiated components were used for analysis.

\section{Definition}

LMR was defined as lymphocyte to monocyte ratio. Percentage of lymph node metastasis was defined as percentage of the patients with metastasis in the whole cohort. Degree of lymphatic metastasis was defined as percentage of the metastatic lymph nodes in all the dissected lymph nodes.

\section{Statistical analysis}

The data were analyzed by SPSS version 20.0 (SPSS Inc, Chicago, IL, USA). Data were presented as mean \pm SD for continuous variables or as counts and percentages for categorical variables. Receiver operating characteristic curve (ROC) was used to determine the cut-off point of LMR. The continuous variables were analyzed with independent $t$-test. Fisher test and $\chi^{2}$ test were used to analyze categorical variables. For non-parametric variables, Mann-Whitney $\mathrm{U}$ test were applied. The variables with significant difference between the two groups were decided by univariate logistic regression. Multivariate logistic regression analysis was performed to determine the correlation between LMR and lymph node metastasis. The odds ratio (OR) with $95 \%$ confidence interval (95\% CI) were obtained. All statistical tests were two-sided. $\mathrm{P}<0.05$ was considered as significant difference. 


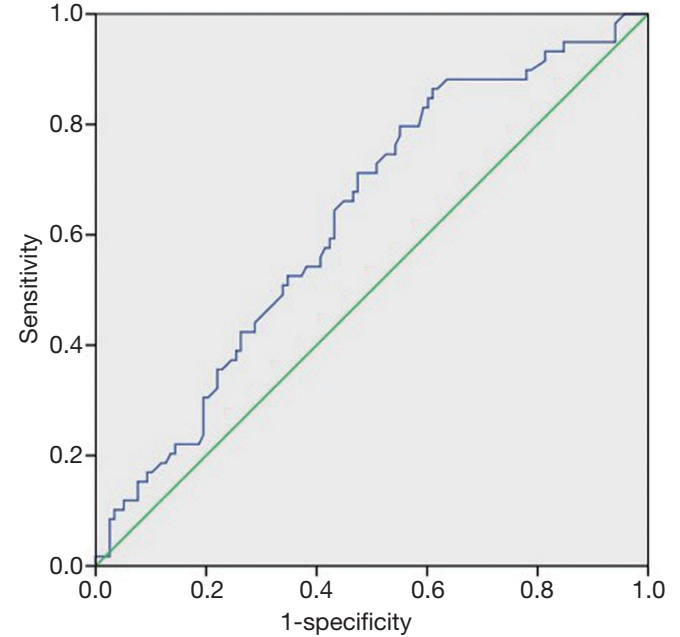

Figure 1 The receiver operating characteristics (ROC) curve.

\section{Results}

\section{Baseline data and LMR of the patients}

A total of 177 subjects (136 males and 41 females; average age of $60.7 \pm 11.7$ years old) met the inclusion criteria and were included in the study. Among them, 117 patients $(117 / 177,66.10 \%)$ had lymph node metastasis.

LMR was used to evaluate the possibility of lymph node metastasis; the area under the curve (AUC) of ROC was 0.629 (Figure 1). The Youden index was maximum with $\mathrm{LMR}=3.79 . \mathrm{LMR}=3.79$ was used as cut-off point; the patients with $L M R \geq 3.79$ were classified as high-LMR group (123 cases) and the patients with LMR $<3.79$ as lowLMR group (54 cases).

In the high-LMR group, there were 70 cases with lymph node metastasis; percentage of lymph node metastasis was $56.9 \%(70 / 123)$. There were 441 positive nodes among 3,749 dissected lymph nodes; degree of lymphatic metastasis was $11.8 \%(441 / 3,749)$. In the low-LMR group, there were 47 cases with lymph node metastasis; percentage of lymph node metastasis was $87.0 \%$ (47/54). There were 534 positive nodes among the 1,548 dissected lymph nodes; degree of lymphatic metastasis was $34.5 \%(534 / 1,548)$. The percentage of lymph node metastasis and the degree of lymphatic metastasis were significantly lower in the highLMR group than those in the low-LMR group.

\section{Comparison of clinicopathological characteristics between the high-LMR group and the low-LMR group}

The statistical results showed that there' no significant difference $(\mathrm{P}>0.05)$ in sex, age, tumor diameter, degree of differentiation, neural invasion, preoperative carcinoembryonic antigen (CEA), carbohydrate antigen-19-9 (CA199) and Ki-67 (Table 1) between those two groups. There's significant difference $(\mathrm{P}<0.05)$ in the tumor site, infiltration depth, lymph node metastasis, number of metastatic lymph nodes $>6$, TNM stage, blood vessel invasion and Lauren's types between high-LMR group and low-LMR group.

\section{Multiple logistic regression analysis for lymph node metastasis}

The factors with significant difference between high-LMR group and low-LMR group were used for multiple logistic regression analysis. LMR was an independent risk factor for lymph node metastasis. The OR value was listed in Table 2.

\section{Discussion}

LMR has been gaining more and more attention as an indicator of inflammatory response with several studies having found that LMR played a protective role in the prognosis of gastric cancer $(12,15)$. In this study, we mainly found that low LMR was positively correlated with lymph node metastasis and lymph node metastasis stage in gastric cancer patient; LMR might have the potential as a preoperative predictor for lymph node metastasis in gastric cancer.

Lymphocyte plays a role in inhibiting tumor occurrence, development, proliferation and distant metastasis $(16,17)$. As reported, tumor infiltrating lymphocyte was highly related with tumor invasion and metastasis $(18,19)$. The statistical results showed that there's significant difference $(\mathrm{P}<0.05)$ in the tumor site, infiltration depth, lymph node metastasis, number of metastatic lymph nodes $>6$, TNM stage, carcinoma cell embolus and Lauren's types. There's no significant difference $(\mathrm{P}>0.05)$ in sex, age, tumor diameter, degree of differentiation, neural invasion, preoperative carcino-embryonic antigen (CEA), carbohydrate antigen-19-9 (CA19-9) and Ki-67. This was in accordance to the previous report (12). It might be attributed to that lower level of lymphocytes may lead to reduced immunological surveillance, and it further decreases lymphocyte mediated anticancer effect (20).

Liu et al. (21) reported that more infiltration of lymphocytes around tumor tissues may bring better prognosis, which depended on the anti-tumor activity of lymphocytes. Further study (22) indicated that cytotoxic 
Table 1 Comparison of clinicopathologic variables between highLMR group and low-LMR group

\begin{tabular}{|c|c|c|c|c|c|}
\hline \multirow[b]{2}{*}{ Variable } & \multirow[b]{2}{*}{$\mathrm{N}$} & \multicolumn{2}{|c|}{ LMR (cases) } & \multirow[b]{2}{*}{$\chi^{2}$} & \multirow[b]{2}{*}{$P$ value } \\
\hline & & $\begin{array}{c}\geq 3.79 \\
(\mathrm{~N}=123)\end{array}$ & $\begin{array}{l}<3.79 \\
(\mathrm{~N}=54)\end{array}$ & & \\
\hline Sex & & & & 0.333 & 0.564 \\
\hline Male & 136 & 96 & 40 & & \\
\hline Female & 41 & 27 & 14 & & \\
\hline Age & & & & 3.366 & 0.067 \\
\hline$<60$ years & 80 & 50 & 30 & & \\
\hline$\geq 60$ years & 97 & 73 & 24 & & \\
\hline Tumor diameter & & & & 3.836 & 0.005 \\
\hline$<5 \mathrm{~cm}$ & 95 & 72 & 23 & & \\
\hline$\geq 5 \mathrm{~cm}$ & 82 & 51 & 31 & & \\
\hline Tumor site & & & & 26.601 & $<0.001$ \\
\hline Preventriculus & 53 & 39 & 14 & & \\
\hline Gastric body & 50 & 13 & 37 & & \\
\hline Gastric antrum & 74 & 47 & 27 & & \\
\hline Differentiation degree & & & & 0.838 & 0.360 \\
\hline High or middle & 44 & 33 & 11 & & \\
\hline Low & 133 & 90 & 43 & & \\
\hline Infiltration depth & & & & 11.992 & 0.001 \\
\hline $\mathrm{T} 1 / \mathrm{T} 2$ & 59 & 51 & 8 & & \\
\hline T3/T4 & 118 & 72 & 46 & & \\
\hline $\mathrm{N}$-staging & & & & 35.423 & $<0.001$ \\
\hline NO & 60 & 53 & 7 & & \\
\hline N1 & 24 & 18 & 6 & & \\
\hline N2 & 41 & 32 & 9 & & \\
\hline N3 & 52 & 20 & 32 & & \\
\hline TNM & & & & 23.409 & $<0.001$ \\
\hline I & 42 & 38 & 4 & & \\
\hline II & 35 & 30 & 5 & & \\
\hline III & 89 & 48 & 41 & & \\
\hline IV & 11 & 7 & 4 & & \\
\hline Blood vessel invasion & & & & 5.168 & 0.023 \\
\hline+ & 102 & 64 & 38 & & \\
\hline- & 75 & 59 & 16 & & \\
\hline
\end{tabular}

Table 1 (continued)
Table 1 (continued)

\begin{tabular}{|c|c|c|c|c|c|}
\hline \multirow[b]{2}{*}{ Variable } & \multirow[b]{2}{*}{$\mathrm{N}$} & \multicolumn{2}{|c|}{ LMR (cases) } & \multirow[b]{2}{*}{$\chi^{2}$} & \multirow[b]{2}{*}{$P$ value } \\
\hline & & $\begin{array}{c}\geq 3.79 \\
(\mathrm{~N}=123)\end{array}$ & $\begin{array}{l}<3.79 \\
(\mathrm{~N}=54)\end{array}$ & & \\
\hline Neural invasion & & & & 0.619 & 0.432 \\
\hline+ & 107 & 72 & 35 & & \\
\hline- & 70 & 51 & 19 & & \\
\hline CEA & & & & 0.068 & 0.795 \\
\hline$\leq 5$ & 143 & 100 & 43 & & \\
\hline$>5$ & 34 & 23 & 11 & & \\
\hline CA199 & & & & 0.082 & 0.774 \\
\hline$<37$ & 146 & 102 & 43 & & \\
\hline$\geq 37$ & 31 & 21 & 10 & & \\
\hline Lauren's type & & & & 9.551 & 0.008 \\
\hline Intestinal type & 68 & 55 & 13 & & \\
\hline Mixed & 42 & 24 & 18 & & \\
\hline Diffused & 67 & 54 & 13 & & \\
\hline Ki-67 & & & & 0.757 & 0.685 \\
\hline$<25 \%$ & 12 & 7 & 5 & & \\
\hline $25-50 \%$ & 44 & 31 & 13 & & \\
\hline$>50 \%$ & 121 & 85 & 36 & & \\
\hline Lymph node metastasis & & & & 15.20 & $<0.001$ \\
\hline No & 60 & 53 & 7 & & \\
\hline Yes & 117 & 70 & 47 & & \\
\hline $\begin{array}{l}\text { Number of metastatic } \\
\text { lymph nodes }\end{array}$ & & & & 33.441 & $<0.001$ \\
\hline$\leq 6$ & 125 & 103 & 22 & & \\
\hline$>6$ & 52 & 20 & 32 & & \\
\hline
\end{tabular}

$\mathrm{T}$ cells and tumor infiltrated $\mathrm{T}$ cells were correlated with lymph node metastasis and survival of gastric cancer. The percentage of lymph node metastasis and the degree of lymphatic metastasis were significantly lower in the highLMR group than those in the low-LMR group. As for $\mathrm{N}$-stage, the percentage of low LMR was significantly higher in N3 group than in N1 group and N2 group. It suggested that low-LMR might mean higher the percentage of lymph node metastasis, the degree of lymphatic metastasis and lymph node stage. The layers of 
Table 2 Odds ratio for lymph node metastasis according to multivariate logistic regression analysis

\begin{tabular}{lcc}
\hline Variable & OR $(95 \% \mathrm{Cl})$ & P value \\
\hline Age $(\geq 60$ years $)$ & $0.915(0.417-2.009)$ & 0.825 \\
Tumor diameter $(\geq 5 \mathrm{~cm})$ & $2.522(1.023-6.219)$ & 0.044 \\
Tumor site & & \\
$\quad$ Gastric body & $0.465(0.161-1.338)$ & 0.156 \\
Gastric antrum & $0.521(0.192-1.414)$ & 0.200 \\
T stage (T3/T4) & $2.017(0.803-5.069)$ & 0.136 \\
Blood vessel invasion & $0.339(0.157-0.729)$ & 0.006 \\
Lauren's type & $1.164(0.432-3.132)$ & 0.764 \\
Mixed & $3.350(1.307-8.587)$ & 0.012 \\
Diffused & $0.229(0.081-0.650)$ & 0.006 \\
\hline LMR & &
\end{tabular}

LMR, lymphocyte to monocyte ratio; OR, odds ratio.

the gastric wall, especially the submucosa and subserosa, are rich in lymphatic duct network, which are favorable for lymphatic metastasis in patients with gastric cancer (23). The decrease of LMR suggests that there's less lymphocyte infiltration in the cancer tissue area, which creates a favorable condition for cancer cells metastasizing to lymph nodes. With the decrease of lymphocyte cells, lymph node metastasis increases, which is in accordance to the "immunological monitoring" theory of B lymphocytes (24). Most studies have shown that increased density of tumorassociated macrophages (TAMs) in tissues can lead to poor prognosis (25). In this study, logistic regression analysis suggested that LMR was an independent predictor of lymph node metastasis.

\section{Conclusions}

There are some limitations in this study, such as small sample size and the nature of retrospective study. In conclusion, low preoperative LMR was positively correlated with lymph node metastasis in gastric cancer patients, and had predictive value for lymph node metastasis in gastric cancer patients. Further study is needed to validate this conclusion.

\section{Acknowledgments}

Funding: None.

\section{Footnote}

Conflicts of Interest: All authors have completed the ICMJE uniform disclosure form (available at http://dx.doi. org/10.21037/tcr.2019.09.17).

Ethical Statement: The authors are accountable for all aspects of the work in ensuring that questions related to the accuracy or integrity of any part of the work are appropriately investigated and resolved. This study was conducted in accordance with the Declaration of Helsinki (as revised in 2013). This study was approved by the ethic committee of Beijing Shijitan Hospital of Capital Medical University. Individual informed consent was waived.

Open Access Statement: This is an Open Access article distributed in accordance with the Creative Commons Attribution-NonCommercial-NoDerivs 4.0 International License (CC BY-NC-ND 4.0), which permits the noncommercial replication and distribution of the article with the strict proviso that no changes or edits are made and the original work is properly cited (including links to both the formal publication through the relevant DOI and the license). See: https://creativecommons.org/licenses/by-nc-nd/4.0/.

\section{References}

1. Somers E. International Agency for Research on Cancer. Encyclopedia of Toxicology 2014;133:1067-9.

2. Song S, Li C, Li S, et al. Derived neutrophil to lymphocyte ratio and monocyte to lymphocyte ratio may be better biomarkers for predicting overall survival of patients with advanced gastric cancer. Onco Targets Ther 2017;10:3145-54.

3. Wagner AD, Grothe W, Haerting J, et al. Chemotherapy in advanced gastric cancer: a systematic review and meta-analysis based on aggregate data. J Clin Oncol 2006;24:2903-9.

4. Deng J, Zhang R, Pan Y, et al. N stages of the seventh edition of TNM Classification are the most intensive variables for predictions of the overall survival of gastric cancer patients who underwent limited lymphadenectomy. Tumor Biol 2014;35:3269-81.

5. Fang WL, Huang KH, Chen JH, et al. Comparison of the survival difference between AJCC 6th and 7th editions for gastric cancer patients. World J Surg 2011;35:2723.

6. Lou N, Zhang L, Chen XD, et al. A novel scoring system associating with preoperative platelet/lymphocyte and 
clinicopathologic features to predict lymph node metastasis in early gastric cancer. J Surg Res 2017;209:153-61.

7. Seevaratnam R, Cardoso R, Mcgregor C, et al. How useful is preoperative imaging for tumor, node, metastasis (TNM) staging of gastric cancer? A meta-analysis. Gastric Cancer 2012;15:S3-18.

8. Huang KH, Lan Y'T, Fang WL, et al. The Correlation between miRNA and Lymph Node Metastasis in Gastric Cancer. Biomed Res Int 2015;2015:543163.

9. Li W, Ye F, Wang D, et al. Protein predictive signatures for lymph node metastasis of gastric cancer. Int J Cancer 2013;132:1851-9.

10. Roxburgh CS and Mcmillan DC. Role of systemic inflammatory response in predicting survival in patients with primary operable cancer. Future Oncol 2010;6:149-63.

11. McMillan DC. Systemic inflammation, nutritional status and survival in patients with cancer. Curr Opin Clin Nutr Metab Care 2009; 12:223-6.

12. Hsu JT, Wang CC, Le PH, et al. Lymphocyte-tomonocyte ratios predict gastric cancer surgical outcomes. J Surg Res 2016;202:284-90.

13. Lu A, Li H, Zheng Y, et al. Prognostic Significance of Neutrophil to Lymphocyte Ratio, Lymphocyte to Monocyte Ratio and Platelet to Lymphocyte Ratio in Patients with Nasopharyngeal Carcinoma. Biomed Res Int 2017;2017:3047802.

14. Japanese Gastric Cancer Association. Japanese gastric cancer treatment guidelines 2010 (ver. 3). Gastric Cancer 2011;14:113-23.

15. Zhou X, Du Y, Xu J, et al. The preoperative lymphocyte to monocyte ratio predicts clinical outcomes in patients with stage II/III gastric cancer. Tumour Biol 2014;35:11659-66.

16. Mantovani A, Allavena P, Sica A, et al. Cancer-related

Cite this article as: Du D, Han Z, Lian D, Amin B, Yan W, Zhang N. The value of preoperative lymphocytes-tomonocytes ratio in predicting lymph node metastasis in gastric cancer. Transl Cancer Res 2019;8(5):2053-2058. doi: 10.21037/ tcr.2019.09.17 inflammation. Nature 2008;454:436-44.

17. Zikos TA, Donnenberg AD, Landreneau RJ, et al. Lung T-cell subset composition at the time of surgical resection is a prognostic indicator in non-small cell lung cancer. Cancer Immunol Immunother 2011;60:819-27.

18. Dunn GP, Old LJ, Schreiber RD. The Immunobiology of Cancer Immunosurveillance and Immunoediting. Immunity 2004;21:137-48.

19. Man YG, Stojadinovic A, Mason J, et al. TumorInfiltrating Immune Cells Promoting Tumor Invasion and Metastasis: Existing Theories. J Cancer 2013;4:84-95.

20. Schreiber RD, Old LJ, Smyth MJ. Cancer immunoediting: integrating immunity's roles in cancer suppression and promotion. Science 2011;331:1565-70.

21. Liu H, Tabuchi T, Takemura A, et al. The granulocyte/ lymphocyte ratio as an independent predictor of tumour growth, metastasis and progression: Its clinical applications. Mol Med Rep 2008;1:699-704.

22. Sharma P, Shen Y, Wen S, et al. CD8 tumor-infiltrating lymphocytes are predictive of survival in muscleinvasive urothelial carcinoma. Proc Natl Acad Sci U S A 2007;104:3967-72.

23. Kojima N, Yonemura Y, Bando E, et al. Optimal extent of lymph node dissection for T1 gastric cancer, with special reference to the distribution of micrometastasis, and accuracy of preoperative diagnosis for wall invasion. Hepatogastroenterology 2008;55:1112-7.

24. Watanabe K, Yasumoto A, Amano Y, et al. Mean platelet volume and lymphocyte-to-monocyte ratio are associated with shorter progression-free survival in EGFR-mutant lung adenocarcinoma treated by EGFR tyrosine kinase inhibitor. PLoS One 2018;13:e0203625.

25. Lin EY, Pollard JW. Role of infiltrated leucocytes in tumour growth and spread. Brit J Cancer 2004;90:2053-8. 